Check for updates

Cite this: RSC Adv., 2019, 9, 21278

Received 18th April 2019

Accepted 25th June 2019

DOI: $10.1039 / c 9 r a 02928 a$

rsc.li/rsc-advances

\title{
Phytochemical composition and the cholinesterase and xanthine oxidase inhibitory properties of seed extracts from the Washingtonia filifera palm fruit $\uparrow$
}

\author{
Sonia Floris, ${ }^{a}$ Antonella Fais, (DD *a Antonella Rosa, (DD ${ }^{\mathrm{b}}$ Alessandra Piras, ${ }^{\mathrm{c}}$ \\ Hanen Marzouki, ${ }^{d}$ Rosaria Medda, (D) a Ana M. González-Paramás, (DD e Amit Kumar, \\ Celestino Santos-Buelga (D) and Benedetta Era ${ }^{a}$
}

\begin{abstract}
The chemical composition and biological properties of palm Washingtonia filifera (Lindl.) $\mathrm{H}$. Wendl. seeds are seldom studied. Bearing this in mind, the seeds of $W$. filifera fruits were analysed for their fatty acid and phenolic composition and their antioxidant activity in addition to their cholinesterase and xanthine oxidase inhibitory activities. Seed extracts were revealed as a good source of phenolics with significant antioxidant activity. The phenolic profile mainly consisted of proanthocyanidins or procyanidin dimers B1-B4 among the major compounds. The highest butyrylcholinesterase inhibitory activity was found in the ethanolic extracts of seeds, with $I_{50}$ values of $13.73 \pm 1.31 \mu \mathrm{g} \mathrm{mL}{ }^{-1}$. Seed alcoholic extracts also displayed interesting xanthine oxidase inhibitory activity, with $I_{50}$ values ranging between $75.2 \pm 17.0 \mu \mathrm{g}$ $\mathrm{mL}^{-1}$ and $95.8 \pm 5.9 \mu \mathrm{g} \mathrm{mL}^{-1}$. Procyanidin B1, a major component in the extracts, could be an important contributor to that activity, as it was found to possess good xanthine oxidase inhibition capacity $\left(I C_{50}\right.$ value of $53.51 \pm 6.03 \mu \mathrm{g} \mathrm{mL}^{-1}$ ). Docking studies were also performed to predict the binding sites of procyanidins B1 and B2 within the xanthine oxidase structure. In all, W. filifera seeds appear as a promising natural source for the extraction of bioactive compounds with antioxidant and butyrylcholinesterase as well as xanthine oxidase inhibitory potential.
\end{abstract}

\section{Introduction}

In recent years, researchers have been attempting to better valorise flora as a natural source of bioactive products. ${ }^{\mathbf{1 , 2}}$ Fruits or underutilized fruit species are receiving more attention, as they contain different phytochemicals that manifest many biological activities, ${ }^{3}$ including the ability to inhibit several enzymatic targets. ${ }^{4,5}$ Moreover, compared to metabolites in the edible portion of fruits, those present in seeds have an added value and good commercial potential as promising phytochemicals/antioxidants for use in food. ${ }^{6}$

The palm family includes a range of plant species with wide application in human food, some of which may also be of

${ }^{a}$ Department of Life and Environmental Sciences, University of Cagliari, Monserrato, CA, Italy.E-mail: fais@unica.it; Tel: +390706754506

${ }^{b}$ Department of Biomedical Sciences, University of Cagliari, Monserrato, CA, Italy ${ }^{c}$ Department of Chemical and Geological Sciences, University of Cagliari, Monserrato, CA, Italy

${ }^{d}$ Laboratory of Transmissible Diseases and Biologically Active Substances, Faculty of Pharmacy, University of Monastir, Tunisia

${ }^{e}$ Grupo de Investigación en Polifenoles (GIP-USAL), Universidad de Salamanca, Spain ${ }^{f}$ Department of Electrical and Electronic Engineering, University of Cagliari, Cagliari, Italy

$\dagger$ Electronic supplementary information (ESI) available. See DOI: 10.1039/c9ra02928a pharmacological interest. ${ }^{7}$ Few studies, however, exist on Washingtonia palms, a genus belonging to the Coryphoideae subfamily that includes two species: W. filifera and W. robusta. Fruits, including the seeds, of $W$. filifera have been analysed for their nutritional composition, with the conclusion that they possess a higher concentration of carbohydrates than proteins. ${ }^{8,9} \mathrm{~W}$. filifera fruits and seeds are also relevant sources of dietary oils. ${ }^{8}$ As for phytochemicals, previously authors ${ }^{10}$ have studied the antioxidant activities of the aerial part of $W$. filifera and reported the presence of eight known flavonoids, including various luteolin and C-glycosyl derivatives, together with two newly described compounds, luteolin 7-O-glucoside $4^{\prime \prime}$-sulfate and 8-hydroxyisoscoparin (i.e., 8hydroxychrysoeriol 6-C-glucoside).

Flavonoids are a major class of the secondary metabolites of plants that occur ubiquitously in foods of plant origin. ${ }^{11-13}$ The greatest antioxidant activity appears to be exhibited by the flavanol class, the procyanidin group..$^{14,15}$

Experimental findings suggest that these molecules can act simultaneously as antioxidants, cholinesterase and xanthine oxidase inhibitors, and anti-fibril agents. ${ }^{16,17}$ Different biological activities have also been reported for C-glycosylated derivatives, such as anti-inflammatory, antioxidant and anticholinesterase properties. ${ }^{18}$

Alzheimer's disease (AD) is a neurodegenerative disease that results from the synaptic dysfunction and death of 
neurons in specific brain regions and circuits, specifically the populations of nerve cells sub-serving memory and cognition. ${ }^{19}$ Cholinesterase inhibitors are in the first line of pharmacotherapy for mild to moderate $\mathrm{AD}$, delaying the breakdown of acetylcholine released into synaptic clefts and enhancing cholinergic neurotransmission. Several studies have been conducted to discover new substances based on plant products that can inhibit the action of cholinesterase and mitigate the effects of $\mathrm{AD}$, while also with fewer side effects than the drugs currently available. ${ }^{20,21}$ The phenomena related to AD are mainly initiated and enhanced by oxidative stress, a process referring to an imbalance between antioxidants and oxidants in favour of oxidants. Several medicinal plants have been shown to assist in mitigating dementia; indeed, different medicinal plants can produce a therapeutic effect owing to different properties, including cholinesterase inhibition and antioxidant activity. ${ }^{22}$

An important enzyme that has been reported to proliferate during oxidative stress is xanthine oxidase (XO), which catalyses the reaction of hypoxanthine to xanthine and xanthine to uric acid. ${ }^{23}$ In both steps, molecular oxygen is reduced, forming the superoxide anion, followed by the generation of hydrogen peroxide. Therefore, compounds that can inhibit xanthine oxidase may reduce both the circulating levels of uric acid and the production of reactive oxygen species (ROS). The overactivity of XO has been associated with the development of gout. ${ }^{24}$

In this context, in search of novel sources of bioactive molecules with potential beneficial effects on $\mathrm{AD}$, the phenolic composition and total polyphenol and flavonoid contents, as well as the antioxidant, anti-cholinesterase and anti-XO properties, have been analysed in pulp and seed extracts from $W$. filifera from two different geographical areas of Tunisia.

\section{Experimental}

\section{Chemicals}

All chemicals were obtained as pure commercial products and used without further purification. Standards of fatty acids and fatty acid methyl esters, Desferal (deferoxamine mesylate salt), Trolox, Folin-Ciocalteau's phenol reagent, 2,2'-azino-bis(3ethylbenzothiazoline-6-sulphonic acid) (ABTS), allopurinol, xanthine oxidase from cow's milk (XO), acetylcholinesterase (AChE) from Electrophorus electricus, butyrylcholinesterase (BChE) from equine serum, xanthine, 5,5'-dithiobis-(2-nitrobenzoic) acid (DTNB), acetylthiocholine iodide (ATCI), $S$-butyrylthiocholine iodide (BTCI), (-)-epicatechin- $(4 \beta, 8)-(+)$-catechin (procyanidin B1) and all solvents used, of the highest available purity, were obtained from Sigma-Aldrich (Milan, Italy). Methanolic $\mathrm{HCl}(3 \mathrm{~N})$ was purchased from Supelco (Bellefonte, PA). The phenolic compound standards were obtained from Extrasynthese (Genay, France). HPLC-grade acetonitrile was obtained from Merck KgaA (Darmstadt, Germany), and formic acid was purchased from Prolabo (VWR International, France). Water was treated in a Milli-Q water purification system (TGI Pure Water Systems, USA).

\section{Plant material}

The fruits of $W$. filifera were collected in Tunisia in the areas of Gabès (G) (33.880444 N, 10.082222 E) and Sousse (S) (35.855727 N, 10.567089 E) in August 2013. The plant was identified by Dr Marzouki Hanene, Laboratory of Transmissible Diseases and Biologically Active Substances, Faculty of Pharmacy, University of Monastir, Tunisia.

The plant materials were washed with deionized water, frozen at $-20{ }^{\circ} \mathrm{C}$ and then lyophilized. Lyophilization was carried out overnight, using an LIO-5P Freeze Dryer apparatus. The dried material was stored at $-20{ }^{\circ} \mathrm{C}$ until required.

The fruits of $W$. filifera, separated as pulp and seeds, were crushed separately and then macerated in different solvent systems to compare the bioactivity of the extracts. The lyophilized plant materials $(25 \mathrm{~g})$ were extracted in $100 \mathrm{~mL}$ of water (AE, aqueous extract), ethanol (EE, ethanol extract) or methanol (ME, methanol extract) for $72 \mathrm{~h}$ at room temperature under continuous stirring. After filtration and centrifugation at $10000 \mathrm{rpm}$, the ethanol and methanol extracts were concentrated, using a rotary evaporator under reduced pressure at 60$70{ }^{\circ} \mathrm{C}$. For fatty acid analysis, the seeds were also extracted with $n$-hexane (HE) in a conventional Soxhlet extraction apparatus, and the samples were further concentrated under vacuum on a rotary evaporator.

Soxhlet extractions were performed using $15 \mathrm{~g}$ of each sample. The powder plant was transferred into a cellulose extraction thimble and inserted into a Soxhlet assembly fitted with a $100 \mathrm{~mL}$ flask. A $50 \mathrm{~mL}$ portion of $n$-hexane was added, and the whole assembly was heated for $6 \mathrm{~h}$ using a heating mantle at $60{ }^{\circ} \mathrm{C}$. The extracts were concentrated using a rotary evaporator at $40{ }^{\circ} \mathrm{C}$, and the dry extracts obtained were stored at $-20{ }^{\circ} \mathrm{C}$ for chemical and biological assays.

\section{Fatty acid analysis of $\boldsymbol{n}$-hexane extracts}

Dried aliquots of seed HE (3 mg) were dissolved in ethanol solution to be subjected to a mild saponification process at room temperature in the dark with a mixture of Desferal/ ascorbic acid/10 N KOH, as previously described..$^{25}$ Dried saponified fractions were injected into an Agilent Technologies 1100 liquid chromatograph equipped with a diode array detector (HPLC-DAD system) (Agilent Technologies, Palo Alto, CA) for the quantification of unsaturated fatty acids (UFAs). The separation was carried out on an Agilent Technologies XDB-C18 Eclipse column $(150 \times 4.6 \mathrm{~mm}, 3.5 \mu \mathrm{m}$ particle size $)\left(\right.$ at $\left.37^{\circ} \mathrm{C}\right)$, equipped with a Zorbax XDB-C18 Eclipse guard column $(12.5 \times$ $4.6 \mathrm{~mm}, 5 \mu \mathrm{m}$ particle size); a mixture of $\mathrm{CH}_{3} \mathrm{CN} / \mathrm{H}_{2} \mathrm{O} / \mathrm{CH}_{3}$ $\mathrm{COOH}(75 / 25 / 0.12, \mathrm{v} / \mathrm{v} / \mathrm{v})$ was used as the mobile phase at a flow rate of $2.3 \mathrm{~mL} \mathrm{~min}{ }^{-1}$, and detection was performed at $200 \mathrm{~nm}^{25}$ UFAs were identified using standard compounds and conventional UV spectra. The quantification of UFAs was made from the peak area ratio, which was based on a calibration curve (in the amount range of $0.5-6 \mu \mathrm{g}$ on the column for PUFA and 1-10 $\mu \mathrm{g}$ for MUFA, respectively) generated from standard compounds 
in $\mathrm{CH}_{3} \mathrm{CN}$ solution. The calibration curves of all the compounds were found to be linear, with correlation coefficients $>0.97$, as previously reported. ${ }^{26}$ An Agilent OpenLAB Chromatography data system was employed to record and integrate the results.

A portion of dried fatty acids after saponification was methylated with $1 \mathrm{~mL}$ of methanolic $\mathrm{HCl}(3 \mathrm{~N})$ for $30 \mathrm{~min}$ at room temperature, as previously described.$^{25}$ Fatty acid methyl esters (FAME) were analysed using a gas chromatograph Hewlett-Packard HP-6890 (Hewlett-Packard, Palo Alto, USA) with a flame ionisation detector (FID) and equipped with a cyanopropyl methyl-polysiloxane HP-23 FAME column $(30 \mathrm{~m}$ $\times 0.32 \mathrm{~mm} \times 0.25 \mu \mathrm{m})\left(\right.$ Hewlett-Packard). ${ }^{25}$ Nitrogen was used as a carrier gas at a flow rate of $2 \mathrm{~mL} \min ^{-1}$. The oven temperature was set to $175^{\circ} \mathrm{C}$; the injector temperature was set to $250{ }^{\circ} \mathrm{C}$; and the detector temperature was set to $300^{\circ} \mathrm{C}$. FAME were identified by comparing the retention times with those of standard compounds and quantified as a percentage of the total amount of fatty acids ( $\mathrm{g} \%$ ) using the Hewlett-Packard software.

\section{Determination of the total phenolics and flavonoids}

The total phenolic and flavonoid content in the extracts were determined as previously reported. ${ }^{25}$ The phenolic concentration was calculated using gallic acid as a reference standard and expressed as $\mathrm{mg}$ of gallic acid equivalents (GAE) per $\mathrm{g}$ of dry weight (dw). Flavonoid concentration was expressed as mg of quercetin equivalents $(\mathrm{QE})$ per $\mathrm{g}$ of $\mathrm{dw}$.

\section{Antioxidant assays and enzyme assays}

ABTS radical-scavenging activity was evaluated with the method reported by Delogu et al. ${ }^{27}$ using Trolox as a standard. Briefly, the free radical $\mathrm{ABTS}^{\cdot+}$ was produced by reacting $7 \mathrm{mM}$ ABTS with $2.45 \mathrm{mM}$ potassium persulfate (final concentration) in aqueous solution and kept in the dark at room temperature for $24 \mathrm{~h}$ before use. Subsequently, an aliquot of this mixture was diluted to obtain an absorbance of approximately $0.7 \pm 0.05$ (mean $\pm \mathrm{SD}$ ). Samples of each extract $(10 \mu \mathrm{L})$ were added to $1 \mathrm{~mL}$ of $\mathrm{ABTS}^{\circ+}$ and the absorbance at $734 \mathrm{~nm}$ was recorded after incubation for $1 \mathrm{~min}$.

The results were expressed as the concentration of sample necessary to cause a $50 \%$ reduction in the original absorbance $\left(\mathrm{EC}_{50}\right)$.

\section{Cholinesterase assay}

Kinetic assays of cholinesterase activity were performed via Ellman's method ${ }^{28}$ with slight modifications ${ }^{29}$ using a plate reader. Briefly, the reaction mixture containing $70 \mu \mathrm{L}$ of phosphate buffer (0.1 M, pH 8.0), enzyme solution (AChE, $4 \mu \mathrm{L}$ or BChE, $6 \mu \mathrm{L}), 100 \mu \mathrm{L}$ of DTNB (1.5 mM final concentration) and inhibitor was dissolved in DMSO at the desired concentrations or in DMSO alone $(2 \%)$. After, ATCI or BTCI $(20 \mu \mathrm{L})$ was added as the substrate to the reaction mixture, and the absorbance was monitored at $405 \mathrm{~nm}\left(37^{\circ} \mathrm{C}\right)$ for 4 minutes. Each extract was evaluated at six concentrations (ranging from 5 to $50 \mu \mathrm{g} \mathrm{mL}{ }^{-1}$ ). Galantamine was used as the standard cholinesterase inhibitor.

\section{Xanthine oxidase assay}

The inhibitory effect of $W$. filifera extracts on xanthine oxidase activity was determined spectrophotometrically by monitoring the formation of uric acid at $295 \mathrm{~nm}$. XO activity was measured according to the method previously reported. ${ }^{5}$ The reaction mixture contained $879 \mu \mathrm{L}$ of $100 \mathrm{mM}$ phosphate buffer $\mathrm{pH} 7.5$, $50 \mu \mathrm{L}$ of an aqueous solution of XO $\left(0.5 \mathrm{U} \mathrm{mL}^{-1}\right)$ and $10 \mu \mathrm{L}$ of the extract sample solution or the control sample solution. After mixing, $61 \mu \mathrm{L}$ of $0.82 \mathrm{mM}$ xanthine solution was added, and the enzyme activity was determined at $295 \mathrm{~nm}$ for $3 \mathrm{~min}$ at $25{ }^{\circ} \mathrm{C}$. Allopurinol was used as the standard XO inhibitor. The inhibition potency for ChEs and XO was expressed as the $\mathrm{IC}_{50}$ values, which represent the inhibitor concentration need to cause $50 \%$ inhibition of enzyme activity. The $\mathrm{IC}_{50}$ values were calculated by interpolation in dose-response curves. The $\mathrm{IC}_{50}$ values displayed represented the mean \pm standard deviation for the three independent assays.

Spectrophotometric determinations were made in an Ultrospec 2100 spectrophotometer (Biochrom Ltd, Cambridge, England) using $1 \mathrm{~cm}$ path cells and with a FLUOstar OPTIMA microplate reader (BMG Labtech, Offenburg, Germany).

\section{HPLC-DAD-ESI/MS analyses}

The methanolic and ethanolic extracts of $W$. filifera fruits were analysed using a Hewlett-Packard 1200 chromatograph (Agilent Technologies, Waldbronn, Germany) equipped with a quaternary pump and a diode array detector (DAD) coupled to an HP Chem Station (rev. A.05.04) data-processing station. The HPLC system was connected via the DAD cell outlet to an API 3200 Qtrap (Applied Biosystems, Darmstadt, Germany) mass spectrometer (MS) consisting of an ESI source and a triple quadrupole-ion trap mass analyzer, which was controlled using Analyst 5.1 software.

Pulp analysis. An Agilent Poroshell 120 EC-C18 $(2.7 \mu \mathrm{m}, 150$ $\times 4.6 \mathrm{~mm}$ ID) thermostated at $35{ }^{\circ} \mathrm{C}$ was used. The solvents were: (A) $0.1 \%$ formic acid and (B) acetonitrile. The elution gradient was performed according to the method previously reported. ${ }^{4}$ Double online detection was carried out in the DAD using 280, 330 and $370 \mathrm{~nm}$ as the preferred wavelengths and in the MS operated in the negative ion mode. Spectra were recorded between $\mathrm{m} / \mathrm{z}=100$ and 1000. Zero-grade air served as the nebulizer gas (30 psi) and as the turbo gas $\left(400^{\circ} \mathrm{C}\right)$ for solvent drying (40 psi). Nitrogen served as the curtain (20 psi) and collision gas (medium). Both quadrupoles were set at unit resolution and EMS and EPI analyses were also performed. The EMS parameters were: ion spray voltage $-4500 \mathrm{~V}, \mathrm{DP}-50 \mathrm{~V}, \mathrm{EP}$ $-6 \mathrm{~V}, \mathrm{CE}-10 \mathrm{~V}$ and cell exit potential (CXP) $-3 \mathrm{~V}$, whereas the EPI settings were: DP $-50 \mathrm{~V}, \mathrm{EP}-6 \mathrm{~V}, \mathrm{CE}-30 \mathrm{~V}$ and CES $10 \mathrm{~V}$.

Seed analysis. An Agilent Poroshell 120 EC-C18 $(2.7 \mu \mathrm{m}, 150$ $\times 4.6 \mathrm{~mm} \mathrm{ID}$ ) at $25{ }^{\circ} \mathrm{C}$ was used. The solvents were: (A) $0.1 \%$ formic acid and (B) acetonitrile. The elution gradient established was isocratic $0-10 \%$ B over $3 \mathrm{~min}, 10-14 \%$ B over $34 \mathrm{~min}$, $14-15 \%$ B over $53 \mathrm{~min}, 15-60 \%$ B over $15 \mathrm{~min}$, isocratic $60 \% \mathrm{~B}$ for $5 \mathrm{~min}$ and re-equilibration of the column, using a flow rate of $0.5 \mathrm{~mL} \mathrm{~min}{ }^{-1}$. Double online detection was carried out as in the DAD using 280, 330 and $370 \mathrm{~nm}$ as the preferred 
wavelengths and in the MS operated in the negative ion mode. Spectra were recorded between $\mathrm{m} / \mathrm{z}=100$ and 1000. Zero-grade air served as the nebulizer gas ( $50 \mathrm{psi}$ ) and as the turbo gas (500 ${ }^{\circ} \mathrm{C}$ ) for solvent drying (40 psi). Nitrogen served as the curtain (25 psi) and collision gas (medium). Both quadrupoles were set at unit resolution and EMS and EPI analyses were also performed. The EMS parameters were: ion spray voltage $-3500 \mathrm{~V}, \mathrm{DP}-65 \mathrm{~V}$, $\mathrm{EP}-10 \mathrm{~V}, \mathrm{CE}-20 \mathrm{~V}$ and cell exit potential (CXP) $-3 \mathrm{~V}$; whereas, the EPI settings were: DP $-40 \mathrm{~V}, \mathrm{EP}-8 \mathrm{~V}, \mathrm{CE}-50$.

The phenolic compounds present in the samples were identified according to their UV and mass spectra and by comparison with commercial standards when available.

\section{Molecular docking}

The crystal structure of the XO (PDB ID: 1FIQ) enzyme was considered as the modeled protein structure for computational investigation. The protein-ligand binding sites were predicted using a COACH-D server, which is an enhanced version of the $\mathrm{COACH}$ server. ${ }^{30}$ The $\mathrm{COACH}$ algorithm predicts ligand poses by using a consensus of five methods. The first four are templatebased methods: TM-SITE, ${ }^{31}$ S-SITE, ${ }^{31}$ COFACTOR $^{32}$ and FINDSITE. ${ }^{33}$ The fifth method is template-free and performs binding site prediction by examining both sequence conservation and the structural geometry of the cavity (region). ${ }^{34}$ The results obtained from the five individual methods were then combined for consensus predictions by the $\mathrm{COACH}$ algorithm. ${ }^{31}$ These ligands were then clustered based on the spatial distance between their geometric centres (average linkage clustering algorithm with a cut-off distance $4 \AA$ A). The final step in the protocol consists of docking the ligand from the user input or the templates into the predicted binding pockets to build their complex structures, employing the molecular docking algorithm AutoDock Vina. ${ }^{35}$ For each predicted binding pocket, up to 10 binding poses are generated, and the one that best matches the consensus prediction of the binding residues is selected.

\section{Statistical analyses}

Statistical differences were evaluated using GraphPad Prism software version 8 (San Diego, CA, USA). Comparison between groups was assessed using the Student's unpaired $t$-test with Welch's correction and by the one-way analysis of variance (oneway ANOVA) followed by the Bonferroni Multiple Comparisons Test. The values with $p<0.05$ were considered significant.

\section{Results and discussion}

Quali-quantitative information on the individual fatty acids (FA) that compose the $W$. filifera $n$-hexane extracts was obtained by GC-FID and HPLC-DAD analyses. Table 1 shows the FA composition by the GC-FID analysis (expressed as \% of total FA, $\mathrm{g} / 100 \mathrm{~g}$ ) of HE obtained from the seeds of $W$. filifera collected in the areas of Sousse and Gabès. The HE Sousse (HES) showed proportions of approximately $60 \%$ saturated FA (SFA) (mainly lauric acid $12: 0$, myristic acid $14: 0$, and palmitic acid $16: 0$, respectively 36,12 , and $6 \%$ ), 28\% monounsaturated FA (MUFA)
Table 1 Fatty acids composition (\% of total fatty acids) obtained by the GC-FID analysis of HE obtained from the seeds of $W$. filifera collected in the areas of Sousse and Gabès ${ }^{a}$

\begin{tabular}{lrr}
\hline & \multicolumn{2}{l}{$\mathrm{g} / 100 \mathrm{~g}$} \\
\cline { 2 - 3 } Fatty acid & \multicolumn{1}{l}{ HES } & \multicolumn{1}{c}{ HEG } \\
\hline $8: 0$ & $1.06 \pm 0.17$ & $1.01 \pm 0.23$ \\
$10: 0$ & $1.55 \pm 0.24$ & $1.61 \pm 0.25$ \\
$12: 0$ & $36.11 \pm 4.23$ & $33.50 \pm 2.70$ \\
$14: 0$ & $12.26 \pm 0.58$ & $10.40 \pm 0.45^{\mathrm{a}}$ \\
$16: 0$ & $6.23 \pm 0.29$ & $6.32 \pm 0.37$ \\
$16: 1$ & $2.23 \pm 0.42$ & $3.07 \pm 0.33^{\mathrm{b}}$ \\
$18: 0$ & $2.62 \pm 0.45$ & $3.05 \pm 0.05$ \\
$18: 1 n-9$ & $25.09 \pm 2.17$ & $25.47 \pm 1.39$ \\
$18: 2 n-6$ & $8.40 \pm 0.63$ & $9.95 \pm 1.36$ \\
$18: 3 n-3$ & $0.04 \pm 0.01$ & $0.06 \pm 0.00$ \\
$18: 3 n-6$ & $0.02 \pm 0.01$ & $0.05 \pm 0.00$ \\
$20: 0$ & $0.33 \pm 0.43$ & $0.08 \pm 0.01$ \\
$20: 1$ & $0.31 \pm 0.06$ & $0.26 \pm 0.19$ \\
SFA & $60.16 \pm 4.03$ & $28.97 \pm 2.92$ \\
MUFA & $27.63 \pm 2.45$ & $10.06 \pm 1.35$ \\
PUFA & $8.47 \pm 0.62$ &
\end{tabular}

${ }^{a}$ Abbreviations: SFA, saturated fatty acids; MUFA, monounsaturated fatty acids; PUFA, polyunsaturated fatty acids. Oil analysis was performed in quadruplicate, and all data are expressed as mean values \pm standard deviations $(\mathrm{SD}) ;(n=4)$. Evaluation of the statistical significance of differences between the two groups was performed using the Student's unpaired $t$-test with Welch's correction; ${ }^{\text {a }} p<0.01$; ${ }^{\mathrm{b}} p<0.05$.

(mainly oleic acid $18: 1 n-9$ and palmitoleic acid $16: 1 n-7$, 25 and $2 \%$, respectively), and $8 \%$ polyunsaturated FA (PUFA), essentially constituted by the essential FA linoleic acid $(18: 2 n$ $-6)$, with traces $(0.04 \%)$ of $\alpha$-linolenic acid $(18: 3 n-3)$. The absolute content of the main UFA was determined by HPLC as follows (Table 2): $304.3 \pm 10.9 \mathrm{mg} \mathrm{g}^{-1}, 102.3 \pm 4.2 \mathrm{mg} \mathrm{g}^{-1}$ and $0.8 \pm 0.04 \mathrm{mg} \mathrm{g}^{-1}$ of $n$-hexane extract; for the acids $18: 1 n-9$, $18: 2 n-6$ and $18: 3 n-3$, respectively.

The HE Gabès (HEG) was characterized by a similar FA profile, with a high level of SFA (56\%, with $34 \%$ of $12: 0$ ), followed by MUFA (29\%) and PUFA (10\%). The HEG showed a slightly lower level of SFA and higher amounts of UFA than HES. Significant differences were only observed in the levels of myristic acid (14:0), with $12 \%$ and $10 \%$ for HES and HEG, respectively $(p<0.01)$, and palmitoleic acid $(16: 1 n-7)$, with $2 \%$ and $3 \%$ for HES and HEG, respectively $(p<0.05)$. The

Table 2 Main unsaturated fatty acids (expressed as $\mathrm{mg} \mathrm{g}^{-1}$ extract), obtained by HPLC analysis, of HE obtained from seeds of $W$. filifera collected in Sousse and Gabès areas ${ }^{a}$

\begin{tabular}{lcc}
\hline Fatty acids & HES & HEG \\
\hline $18: 1 n-9$ & $304.33 \pm 10.93$ & $275.41 \pm 13.26$ \\
$18: 2 n-6$ & $102.31 \pm 4.21$ & $108.19 \pm 6.26$ \\
$18: 3 n-3$ & $0.84 \pm 0.04$ & $0.88 \pm 0.06$
\end{tabular}

${ }^{a}$ Oil analysis was performed in quadruplicate and all data are expressed as mean values \pm standard deviations $(\mathrm{SD}) ;(n=4)$. 
absolute values of the main UFA determined by HPLC (Table 2) for the HEG were $275.4 \pm 13.3 \mathrm{mg} \mathrm{g}^{-1}, 108.2 \pm 6.3 \mathrm{mg} \mathrm{g}^{-1}$ and $0.9 \pm 0.1 \mathrm{mg}$ g; for acids $18: 1 n-9,18: 2 n-6$ and $18: 3 n-3$, respectively. Both HES and HEG contained lauric acid (12:0) as the main fatty acid (34-36\%) but also exhibited a high content of oleic acid (18: $1 n-9 ; 25 \%)$. Thus, $W$. filifera seed oil can be regarded as a lauric-oleic oil because of the abundance of these two fatty acids. ${ }^{8}$

The FA composition of the HEG and HES oil extracts determined herein was slightly different from that of $W$. filifera seed oil obtained previously from a Tunisian sample. ${ }^{8}$ Specifically, like the HEG and HES, the major FA were SFA (43\%), followed by MUFA (41\%) and PUFA (16\%); however, Tunisian seed oil showed oleic acid as the most abundant fatty acid (41\%), followed by lauric acid (18\%), linoleic acid (16\%), myristic acid (11\%) and palmitic acid (9\%). This result could be ascribable to several factors, e.g., differences in FA metabolism due to the impact of the harvesting location such as climate, soil, and water availability.

The total phenolic and flavonoid contents in the analysed seed extracts are shown in Table 3. The highest total phenolic content was found in ME, followed by EE and AE. MEG showed a total phenolic content two times higher than the corresponding EEG. Very low amounts of phenolic compounds were also detected in the pulp extracts (data not shown). A positive correlation was found between total phenolic content versus flavonoid content $\left(r=0.98, r^{2}=97 \%\right)$, determined in the alcoholic seed extracts, whereas hardly any flavonoids were found in the aqueous extracts.

Phenolic compounds have redox properties, which allow them to act as antioxidants. ${ }^{36}$ The antioxidant activity of the extracts was assessed by their ability to scavenge the ABTS radical. The results obtained for the seed extracts are included in Table 3. As for the total phenolic content, the aqueous extracts showed lower antioxidant capacity (higher EC $_{50}$ values) than the alcoholic extracts. The correlation of the total phenol content and ABTS radical scavenging activity was also shown in Fig. S1 (ESI†). This correlation seems logical considering that the Folin-Ciocalteu reagent measures the reducing capacity of a sample, lacking specificity for phenolics. Pulp extracts presented much lower antioxidant capacity than seeds, with the $\mathrm{EC}_{50}$ value for
ABTS radical scavenging ability was higher than $150 \mu \mathrm{g}$ $\mathrm{mL}^{-1}$, which is in line with their low levels of phenolic compounds.

The characterization of individual phenolic compounds was performed by HPLC-DAD/ESI-MS. Data of the retention time, $\lambda_{\max }$, pseudomolecular ions, main fragment ions in $\mathrm{MS}^{2}$, and tentative identification are presented in Table 4. As can be seen, the sample mostly consists of flavan-3-ols (i.e., catechins and proanthocyanidins). Epicatechin and procyanidin B1 were identified by comparison with standards, whereas the identities of the procyanidin dimers B2-B4 and trimer $\mathrm{C} 2$ were tentatively assigned by comparison with data available in our data library. The identities of the remaining compounds were established based on their molecular weights. A point to highlight is the presence of some proanthocyanidins containing possible (epi)afzelechin units as well as A-type linkages. B-type procyanidin dimers (B1B4) were among the main phenolic compounds in the extracts of $W$. filifera seeds that, in a previous study, were reported for their different biological activity. ${ }^{37,38}$ Minor amounts of other flavonoids, mainly quercetin and isorhamnetin derivatives possessing sulfate residues, were also detected. Although flavonoid sulfates are not very common in plants, they have been reported to occur in species of the Palmae family. ${ }^{39}$ As far as we know, no previous reports have been published on the phenolic profile of $W$. filifera seeds.

The anticholinesterase activity of all the extracts at a concentration of $20 \mu \mathrm{g} \mathrm{mL}{ }^{-1}$ was checked using AChE/BChE assays.

Table 5 shows the AChE and BChE inhibitory activities of the of $W$. filifera seeds extracts, compared with those of the standard inhibitor galantamine. The $\mathrm{IC}_{50}$ for AChE was not determined because the inhibition at the highest screened concentration $\left(20 \mu \mathrm{g} \mathrm{mL}^{-1}\right)$ was less than $40 \%$.

The $\mathrm{IC}_{50}$ values ranged from $13.73 \pm 1.31 \mu \mathrm{g} \mathrm{mL} \mathrm{mL}^{-1}$ to $27.30 \pm$ $5.37 \mu \mathrm{g} \mathrm{mL} \mathrm{m}^{-1}$ in the different seed extracts. There was no statistically significant difference between the $\mathrm{IC}_{50}$ values of EEG, MEG and AEG compared to galantamine. The results obtained revealed that EEG showed very potent BChE inhibitory activity, with $\mathrm{IC}_{50}$ values $\left(13.73 \pm 1.31 \mu \mathrm{g} \mathrm{mL} \mathrm{mL}^{-1}\right)$ close to those of the standard drug galantamine $\left(\mathrm{IC}_{50}=7.65 \pm 1.78 \mu \mathrm{g} \mathrm{mL} \mathrm{m}^{-1}\right)$ calculated under the same experimental conditions.

Table 3 Total phenolic and flavonoid contents, ABTS radical scavenging activity in $W$. filifera seed extracts

\begin{tabular}{lllr}
\hline & Total Phenolic mg GAE per g dw & Flavonoid mg QE per g dw $^{\text {ABTS EC }_{50} \mu g \mathrm{~mL}^{-1}}$ \\
\hline EEG & $325.96 \pm 32.20^{\mathrm{a}, \mathrm{b}}$ & $215.43 \pm 98.61^{\mathrm{a}}$ & $11.11 \pm 1.15^{\mathrm{a}, *}$ \\
EES & $412.30 \pm 115.78^{\mathrm{a}, \mathrm{b}}$ & $308.33 \pm 137.23^{\mathrm{a}}$ & $9.06 \pm 0.35^{\mathrm{a}, *}$ \\
MEG & $708.83 \pm 169.10^{\mathrm{a}}$ & $591.98 \pm 386.14^{\mathrm{a}}$ & $5.52 \pm 0.84^{\mathrm{b}}$ \\
MES & $637.4 \pm 275.11^{\mathrm{a}, \mathrm{c}}$ & $462.60 \pm 294.20^{\mathrm{a}}$ & $9.71 \pm 1.21^{\mathrm{a}, *}$ \\
AEG & $133.54 \pm 30.0^{\mathrm{b}}$ & $\S$ & $22.64 \pm 0.14^{\mathrm{c}, *}$ \\
AES & $233.06 \pm 33.68^{\mathrm{b}, \mathrm{c}}$ & & $17.78 \pm 0.45^{\mathrm{d}, *}$ \\
Trolox & & & $3.4 \pm 0.3$
\end{tabular}

Each value is the mean $\pm \mathrm{SD}$ of three independent measurements $(n=3) .{ }^{\S}$ Below limit of detection. ${ }^{\mathrm{a}, \mathrm{b}, \mathrm{c}, \mathrm{d}}$ Different letters within the same column denote statistically significant differences between extracts $(p<0.05)$. *Values of EC $_{50}$ of EEG, EES, MES, AEG and AES compared to Trolox are significantly different $(p<0.01)$. 
Table 4 Retention time $\left(R_{\mathrm{t}}\right)$, wavelengths of maximum absorption $\left(\lambda_{\max }\right)$, mass spectral data, and tentative identification of phenolic compounds detected in $W$. filifera seeds

\begin{tabular}{|c|c|c|c|c|c|}
\hline Peak & $R_{\mathrm{t}}(\min )$ & $\lambda_{\max }(\mathrm{nm})$ & $\begin{array}{l}\text { Pseudomolecular } \\
\text { ion }[\mathrm{m}-\mathrm{H}]^{-}(\mathrm{m} / \mathrm{z})\end{array}$ & $\operatorname{MS}^{2}(m / z)$ & Tentative identification \\
\hline 1 & 12.0 & 260,293 & 331 & & Galloylglucose \\
\hline 3 & 20.2 & 279 & 577 & $451,425,407,289$ & B-type procyanidin dimer (B3) \\
\hline 4 & 20.7 & 279 & 577 & & B-type procyanidin dimer (B1) \\
\hline 5 & 21.1 & 278 & 865 & $695,577,425,407,287$ & B-type procyanidin trimer (C2) \\
\hline 8 & 23.7 & & 863 & & A-type procyanidin trimer \\
\hline 9 & 25.4 & & 1153 & $849,577,407,287$ & B-type procyanidin tetramer \\
\hline 10 & 27.5 & 278 & 289 & $245,203,179,109$ & Epicatechin \\
\hline 11 & 28.4 & & 561 & $435,407,289$ & (epi)Catechin-(epi)afzelechin dimer \\
\hline 12 & 29.4 & 283 & 449 & 287,269 & Dihydrokaempferol hexoside \\
\hline 13 & 30.9 & & 863 & $711,575,423$ & A-type procyanidin trimer \\
\hline 18 & 40.5 & & 865 & & B-type procyanidin trimer \\
\hline 19 & 41.4 & & 849 & $697,577,407,287$ & $\begin{array}{l}\text { B-type proanthocyanidin trimer } \\
\text { containing one afzelechin unit }\end{array}$ \\
\hline 20 & 43.3 & 254,353 & 689 & 301 & Quercetin rutinoside sulfate \\
\hline 21 & 43.8 & & 1441 & & B-type procyanidin pentamer \\
\hline 22 & 45.3 & 256,358 & 703 & 315 & Isorhamnetin rutinoside sulfate \\
\hline 23 & 45.3 & & 577 & & B-type procyanidin dimer \\
\hline 24 & 46.5 & & 849 & & $\begin{array}{l}\text { B-type procyanidin trimer containing one } \\
\text { afzelechin unit }\end{array}$ \\
\hline 26 & 50.4 & & 557 & 315 & Isorhamnetin glucoside sulfate \\
\hline 28 & 61.8 & 255,353 & 463 & 301 & Quercetin glucoside \\
\hline 29 & 65.1 & & 577 & & B-type procyanidin dimer \\
\hline
\end{tabular}

This can be considered a satisfactory result, since the standard inhibitor is a single molecule, while a mixture of numerous compounds exists in the plant extracts.

The inhibitory activity against BChE of a procyanidin B1 standard at a concentration of $20 \mu \mathrm{g} \mathrm{mL} \mathrm{m}^{-1}$ was also checked, obtaining a value of $18.98 \pm 2.52 \%$.

Thus, anti-BChE activity observed in the seed extracts cannot be mainly attributed to procyanidin B1, even if it is present in high concentrations. However, our findings led us to consider that this compound could contribute to the anti-BChE effect in these extracts.

Several studies on plant AChE inhibitors have been performed $;^{40}$ however, fewer BChE inhibitors have been identified. ${ }^{41}$ No ChE inhibitory activity was found for any of the pulp extracts examined (data not shown).

ChEs inhibition has been extensively used as an approach for the treatment of Alzheimer's disease (AD). BChE activity progressively increases in patients with $\mathrm{AD}$, while $\mathrm{AChE}$ activity

Table 5 Percentage of inhibition (\% I) at $20 \mu \mathrm{g} \mathrm{mL}^{-1}$ and the $\mathrm{IC}_{50}$ value $\left(\mu \mathrm{g} \mathrm{mL}^{-1}\right.$ ) of $W$. filifera seeds extracts against cholinesterases

\begin{tabular}{|c|c|c|c|c|}
\hline Extracts & AChE\% I & $\mathrm{AChE} \mathrm{IC}_{50}$ & BChE\% I & $\mathrm{BChE} \mathrm{IC}_{50}$ \\
\hline EEG & $3.2 \pm 0.5$ & n.d & $65.6 \pm 0.78$ & $13.73 \pm 1.31^{\mathrm{a}}$ \\
\hline EES & $16.5 \pm 6.22$ & n.d & $53.9 \pm 6.5$ & $27.30 \pm 5.37^{\mathrm{b}, *}$ \\
\hline MES & $20.9 \pm 1.56$ & n.d & $63.1 \pm 1.91$ & $22.6 \pm 2.72^{\mathrm{b}, \mathrm{c}, *}$ \\
\hline AEG & $28.6 \pm 7.78$ & n.d & $45.6 \pm 1.06$ & $15.08 \pm 1.05^{\mathrm{a}, \mathrm{c}}$ \\
\hline AES & $38.5 \pm 11.6$ & n.d & $48.5 \pm 1.06$ & $18.51 \pm 0.001^{\mathrm{a}, \mathrm{c}, *}$ \\
\hline
\end{tabular}

n.d: not determined because the inhibition at the highest screened concentration $\left(20 \mu \mathrm{g} \mathrm{mL} \mathrm{L}^{-1}\right)$ was less than $40 \%$. Values were expressed as mean \pm $\mathrm{SD}(n=3) .{ }^{\mathrm{a}, \mathrm{b}, \mathrm{c}}$ Different letters within the same column denote statistically significant differences between extracts $(p<0.05) .{ }^{*} \mathrm{Values}$ of the IC $\mathrm{C}_{50}$ of EES, MES and AES compared to galantamine are significantly different $(p<0.05)$. 
Table 6 Percentage of inhibition (\% I) at $150 \mu \mathrm{g} \mathrm{mL}^{-1}$, the $\mathrm{IC}_{50}$ value ( $\mu \mathrm{g} \mathrm{mL}^{-1}$ ) and the inhibitory mode of $W$. filifera seeds extracts against xanthine oxidase ${ }^{a}$

\begin{tabular}{llll}
\hline Extracts & $\% \mathrm{I}$ & $\mathrm{IC}_{50}$ & Inhibitory mode \\
\hline EEG & $52.4 \pm 0.8$ & $95.8 \pm 5.9^{*}$ & Mixed \\
EES & $63.9 \pm 0.1$ & $87.0 \pm 0.5^{*}$ & Mixed \\
MEG & $72.8 \pm 0.3$ & $75.2 \pm 17.0^{*}$ & Mixed \\
MES & $74.6 \pm 0.2$ & $76.1 \pm 5.2^{*}$ & Mixed \\
AEG & $36.7 \pm 0.1$ & n.d & n.d \\
AES & $37.7 \pm 0.1$ & n.d & n.d \\
Allopurinol & & $2.0 \pm 0.4$ &
\end{tabular}

${ }^{a}$ n.d: not determined because inhibition at the highest screened concentration $\left(150 \mu \mathrm{g} \mathrm{mL} \mathrm{mL}^{-1}\right)$ was less than $40 \%$. Values were expressed as mean $\pm \mathrm{SD}(n=3)$. Values of the $\mathrm{IC}_{50}$ for alcoholic extracts compared to allopurinol were significantly different $(p<0.05)$.

remains unchanged or declines. Therefore, the use of molecules selectively interacting with BChE might have a relevant role in the treatment of patients with advanced $\mathrm{AD}$.

The extracts of $W$. filifera were proven to have great potential and should be considered in future studies to identify the constituents responsible for the selective BChE inhibitory activity.

The extracts were also evaluated for their inhibition of XO enzyme activity (Table 6).

It was encouraging to observe that only pulp extracts were inactive against the XO enzyme, while all seed extracts displayed inhibitory activity at $150 \mu \mathrm{g} \mathrm{mL} \mathrm{m}^{-1}$, ranging between $36.7 \pm 0.1$ and $74.6 \pm 0.2 \%$. The alcoholic seed extracts showed $\mathrm{IC}_{50}$ values for the XO inhibitory activity in the $75.2 \pm 17.0 \mu \mathrm{g} \mathrm{mL} \mathrm{m}^{-1}$ and $95.8 \pm 5.9 \mu \mathrm{g} \mathrm{mL}^{-1}$ range, higher than those of the standard drug allopurinol $\left(\mathrm{IC}_{50}=2.0 \pm 0.4 \mu \mathrm{g} \mathrm{mL}{ }^{-1}\right)$.

Flavan-3-ols, the major compounds in the seed extracts, have been reported to possess inhibitory XO activity. ${ }^{\mathbf{4 2 , 4 3}}$ Epicatechin behaves as a good XO inhibitor. ${ }^{44}$ Procyanidin B1, one the major components detected in the seed extracts of $W$. filifera, also revealed good XO inhibition capacity, showing an $\mathrm{IC}_{50}$ value of $53.5 \pm 6.0 \mu \mathrm{g} \mathrm{mL}^{-1}$. As far as we know, no previous reports exist on XO inhibition by this procyanidin.

Molecular docking is a powerful technique that allows the prediction and identification of the most probable binding mode of the ligand to a partner protein. ${ }^{44}$ Therefore, to predict the best ligand pose within the XO binding site, we performed the docking of ligands procyanidin B1 and B2, which consists of catechin and epicatechin units joined in a beta-configuration. For comparison, the docking of ligands catechin and epicatechin, which are the elementary flavan-3-ol units in these dimers, was also checked. ${ }^{45}$

For the procyanidin ligands (B1, B2), the two most probable binding sites (Fig. 1) was observed. Binding site 1, which is located a distance from the protein active site exhibited the best docking energy values for both ligands (Table 7). We observed a reasonable overlap in the ligand poses, with procyanidin B1 displaying favourable docking energy.

On the other hand, binding site 2 (Fig. 1) for both procyanidin ligands (B1, B2) was found to be near the XO protein active site. Interestingly, the binding region for the ligands

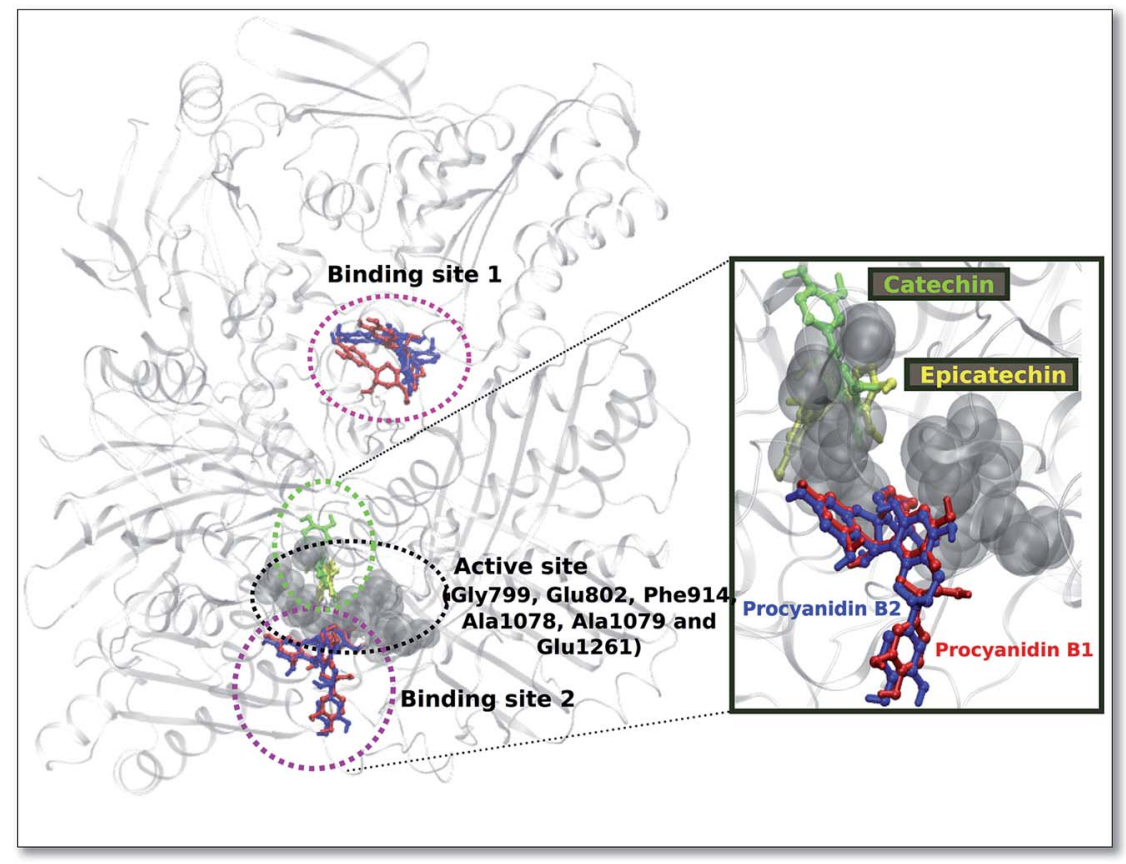

Fig. 1 Predicted docked positions for the ligands bound to the XO protein. The active site residues are shown as grey van der Waals spheres (grey). Two probable binding sites 1 and 2 for the ligands procyanidin B1 (red) and B2 (blue) are circled in pink, while the binding region for the ligands catechin (green) and epicatechin (yellow) are within the green circle. In the rectangular box, a zoomed representation of the binding region for the ligands close to the active site is shown. 
Table 7 Summary of the predicted docking energies for ligands bound to $\mathrm{XO}$. In column 3, the confidence score (C-score) of the predicted binding residues, associated with specific ligand-binding clusters are shown. In column 4, we reported the cluster size that represented the population number of ligand structures specific to a binding site

\begin{tabular}{llll}
\hline Protein-ligand & Docking energy $\left(\mathrm{kcal} \mathrm{mol}^{-1}\right)$ & C-score & Cluster size \\
\hline XO-procyanidin B1 & -4.6 (site 1) & 0.12 & 28 \\
& $-2.7($ site 2) & 0.22 & 41 \\
XO-procyanidin B2 & $-3.8($ site 1) & 0.12 & 28 \\
& $-3.0($ site 2) & 0.22 & 41 \\
XO-catechin & $-8.6 \mathrm{kcal} \mathrm{mol}^{-1}$ & 0.19 & 30 \\
XO-epicatechin & $-9.2 \mathrm{kcal} \mathrm{mol}^{-1}$ & 0.15 & 28
\end{tabular}

catechin and epicatechin are in the active site and are in close proximity to binding site 2 of the procyanidin ligands.

Experimental data performed on the seed extracts indicated a mixed-type inhibition against the XO-enzyme. Now, considering that the concentration of procyanidin is pronounced (among the dimers) in the seed extracts, a plausible explanation for the mixed-type inhibition can be established from the spatial location of the predicted binding sites

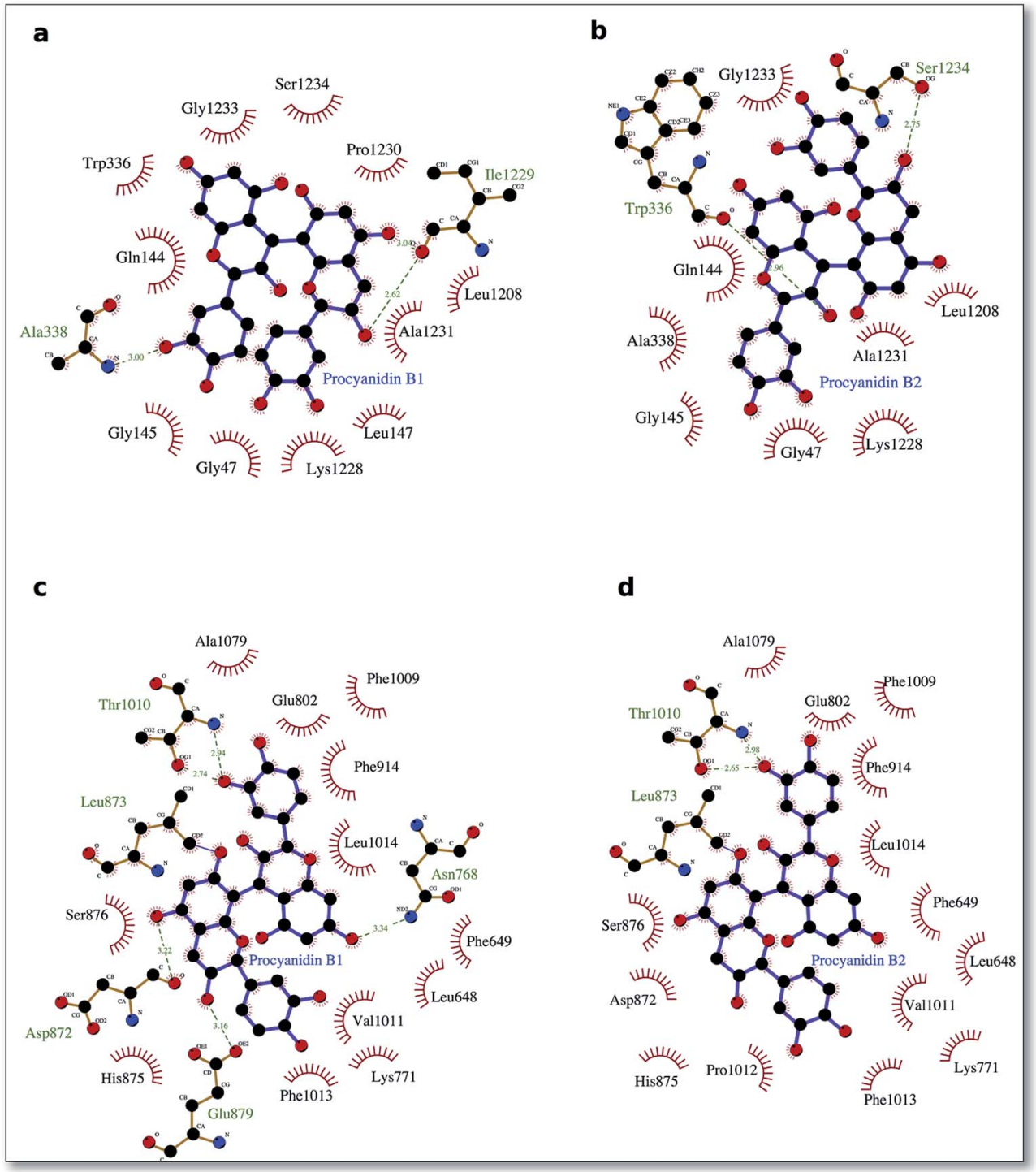

Fig. 2 Procyanidin ligands B1 and B2 bound to the XO protein. In (a) and (b), the ligand poses in the binding site 1, while in (c) and (d), it does so in the binding site 2 . Hydrophobic interactions are represented by red spokes radiating towards the interacting ligand atoms, while hydrogenbonded interactions are shown with a dashed green line. 


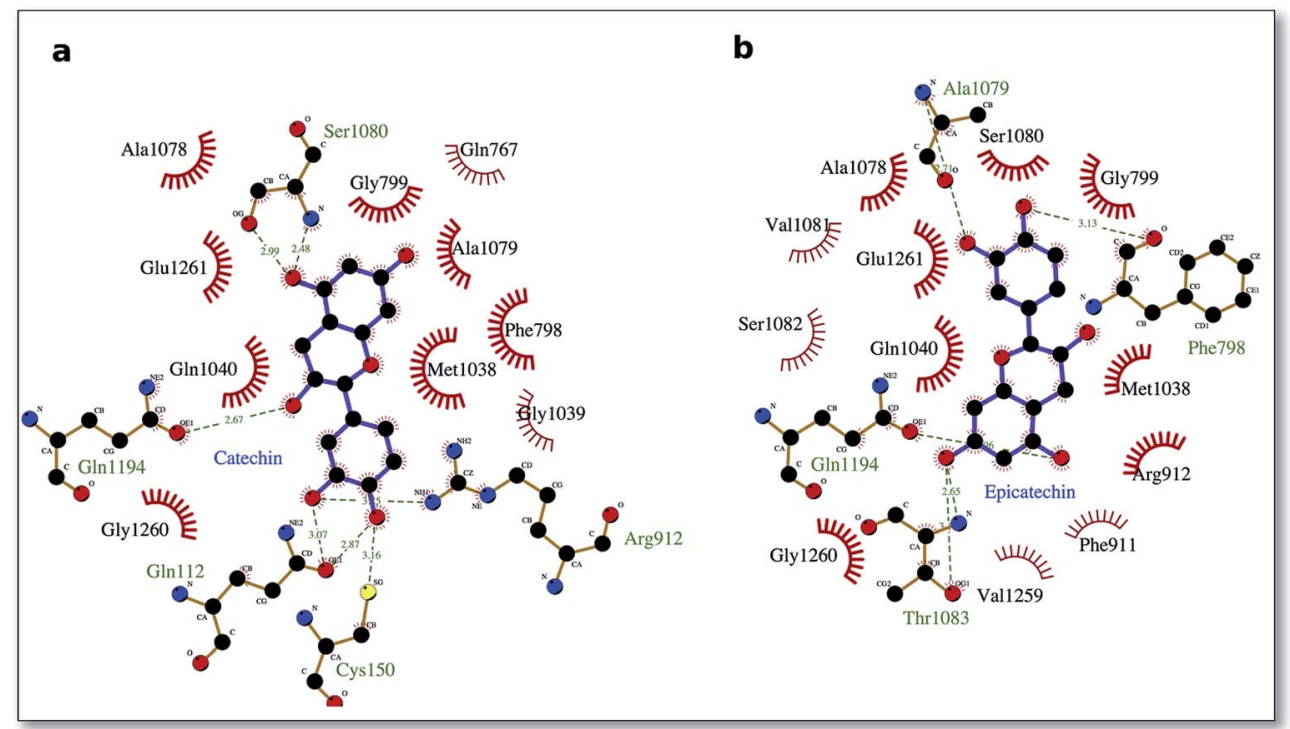

Fig. 3 Interaction picture of the ligands catechin and epicatechin bound to the XO protein. Hydrophobic interactions are represented by red spokes radiating towards the interacting ligand atoms, while hydrogen-bonded interactions are shown with a dashed green line.

(different from the active site) for the procyanidin ligands. Since experiments revealed very low inhibitory activity for procyanidin B1 against BChE, we do not discuss the docking results of procyanidin $\mathrm{B} 1$ and the $\mathrm{BChE}$ protein. However, for completeness, we have provided the data in the ESI. $\dagger$

To delve into the binding mode of the ligands with the XO protein, further examination was made using Ligplot software, ${ }^{46}$ which revealed a conserved interaction image regarding XO binding for both procyanidins B1 and B2 (Fig. 2) and for catechin and epicatechin (Fig. 3).

For binding site 1 (Fig. 2a and b), we note two additional interactions involving residues Leu 147, Ile 1229 and Pro 1230 and procyanidin $\mathrm{B} 1$, thus confirming the better docking energy value with respect to procyanidin B2. On the other hand, for binding site 2, a good overlap between the ligands poses was confirmed by a conserved interaction picture (Fig. $2 \mathrm{c}$ and d).

The well-conserved binding regions for the ligands catechin and epicatechin (Fig. 3), involving interactions with amino acid residues Gly799, Glu802, Phe914, Ala1078, Ala1079 and Glu1261 in the active site, confirmed the significantly better docking energy with respect to the procyanidin ligands (B1, B2).

\section{Conclusion}

Natural products from plants provide unlimited opportunities for new drugs because their chemical diversity shows a range of biological activities. W. filifera seeds have been revealed to possess components with antioxidant and also butyrylcholinesterase and xanthine oxidase inhibition properties, which is of interest, considering that they are an inedible part of the fruit and are usually discarded. Molecular docking studies predicted the XO protein binding regions of procyanidin ligands and provided a plausible explanation to the mixed-type inhibition characteristic found for the seed extracts against the
XO enzyme. These findings should contribute to valorise $W$. filifera seeds as a source for the extraction of bioactive compounds with nutraceutical and therapeutic potential.

\section{Conflicts of interest}

There are no conflicts to declare.

\section{Acknowledgements}

The authors are grateful to Dr Giorgia Sarais (University of Cagliari) for her support. This work was supported by the University of Cagliari. The GIP-USAL was financially supported by MINECO (Spanish National Projects AGL2015-64522-C2).

\section{References}

1 M. G. Arvizu-Espinosa, G. L. von Poser, A. T. Henriques, A. Mendoza-Ruiz, A. Cardador-Martínez, R. Gesto-Borroto, P. N. Núñez-Aragón, M. L. Villarreal-Ortega, A. Sharma and A. Cardoso-Taketa, J. Nat. Prod., 2019, 82, 785-791.

2 B. David, J.-L. Wolfender and D. A. Dias, Phytochem. Rev., 2014, 14, 299-315.

3 R. K. Firdose, Afr. J. Pharm. Pharmacol, 2011, 5, 2067-2072.

4 A. Di Petrillo, A. M. González-Paramás, B. Era, R. Medda, F. Pintus, C. Santos-Buelga and A. Fais, BMC Complement Altern. Med., 2016, 16, 453.

5 A. Fais, B. Era, A. Di Petrillo, S. Floris, D. Piano, P. Montoro, C. I. G. Tuberoso, R. Medda and F. Pintus, BioMed Res. Int., 2018, 2018, 1-9.

6 Y. Xu, M. Fan, J. Ran, T. Zhang, H. Sun, M. Dong, Z. Zhang and H. Zheng, Saudi J. Biol. Sci., 2016, 23, 379-388.

7 P. Geavlete, R. Multescu and B. Geavlete, Ther. Adv. Urol., 2011, 3, 193-198. 
8 I. A. Nehdi, Food Chem., 2011, 126, 197-202.

9 J. W. Cornett, Principes, 1987, 31, 159-161.

10 N. H. El-Sayed, N. M. Ammar, S. Y. Al-Okbi, L. T. A. ElKassem and T. J. Mabry, Nat. Prod. Res., 2006, 20, 57-61.

11 P. C. Hollman and M. B. Katan, Biomed. Pharmacother., 1997, 51, 305-310.

12 T. R. Dias, M. G. Alves, S. Casal, P. F. Oliveira and B. M. Silva, Curr. Med. Chem., 2017, 24, 334-354.

13 A. Tresserra-Rimbau, R. M. Lamuela-Raventos and J. J. Moreno, Biochem. Pharmacol., 2018, 156, 186-195.

14 C. A. Rice-Evans and N. J. Miller, Biochem. Soc. Trans., 1996, 24, 790-795.

15 J. E. Wood, S. T. Senthilmohan and A. V. Peskin, Food Chem., 2002, 77, 155-161.

16 D. Szwajgier, Pol. J. Food Nutr. Sci., 2014, 64, 59-64.

17 D. Di Majo, M. La Guardia, G. Leto, M. Crescimanno, C. Flandina and M. Giammanco, Int. J. Food Sci. Nutr., 2014, 65, 886-892.

18 J. S. Choi, M. N. Islam, M. Y. Ali, Y. M. Kim, H. J. Park, H. S. Sohn and H. A. Jung, Arch. Pharmacal Res., 2014, 37, 1354-1363.

19 G. M. Shankar and D. M. Walsh, Mol. Neurodegener., 2009, 4, 48.

20 N. SatheeshKumar, P. K. Mukherjee, S. Bhadra and B. P. Saha, Phytomedicine, 2010, 17, 292-295.

21 M. Herrera-Ruiz, G. García-Morales, A. Zamilpa, M. González-Cortazar, J. Tortoriello, E. Ventura-Zapata and E. Jimenez-Ferrer, Bol. Latinoam. Caribe Plantas Med. Aromat., 2012, 11, 526-541.

22 T. S. Anekonda and P. H. Reddy, Brain Res. Rev., 2005, 50, 361-376.

23 R. Harrison, Drug Metab. Rev., 2004, 36, 363-375.

24 B. T. Emmerson and A. J. J. Wood, N. Engl. J. Med., 1996, 334, 445-451.

25 A. Rosa, A. Maxia, D. Putzu, A. Atzeri, B. Era, A. Fais, C. Sanna and A. Piras, Food Chem., 2017, 230, 82-90.

26 A. Rosa, D. Caprioglio, R. Isola, M. Nieddu, G. Appendino and A. M. Falchi, Food Funct., 2019, 10, 1629-1642.

27 G. L. Delogu, M. J. Matos, M. Fanti, B. Era, R. Medda, E. Pieroni, A. Fais, A. Kumar and F. Pintus, Bioorg. Med. Chem. Lett., 2016, 26, 2308-2313.
28 G. L. Ellman, K. D. Courtney, V. Andres and R. M. Featherstone, Biochem. Pharmacol., 1961, 7, 88-95.

29 A. Kumar, F. Pintus, A. Di Petrillo, R. Medda, P. Caria, M. J. Matos, D. Vina, E. Pieroni, F. Delogu, B. Era, G. L. Delogu and A. Fais, Sci. Rep., 2018, 8, 4424.

30 Q. Wu, Z. Peng, Y. Zhang and J. Yang, Nucleic Acids Res., 2018, 46, W438-W442.

31 J. Yang, A. Roy and Y. Zhang, Bioinformatics, 2013, 29, 25882595.

32 A. Roy, J. Yang and Y. Zhang, Nucleic Acids Res., 2012, 40, W471-W477.

33 M. Brylinski and J. Skolnick, Proc. Natl. Acad. Sci. U. S. A., 2008, 105, 129-134.

34 J. A. Capra, R. A. Laskowski, J. M. Thornton, M. Singh and T. A. Funkhouser, PLoS Comput. Biol., 2009, 5, e1000585.

35 O. Trott and A. J. Olson, J. Comput. Chem., 2010, 31, 455-461. 36 M. A. Soobrattee, V. S. Neergheen, A. Luximon-Ramma, O. I. Aruoma and T. Bahorun, Mutat. Res., Fundam. Mol. Mech. Mutagen., 2005, 579, 200-213.

37 Z.-Q. Ling, B.-J. Xie and E.-L. Yang, J. Agric. Food Chem., 2005, 53, 2441-2445.

38 C. Ao, T. Higa, H. Ming, Y.-t. Ding and S. Tawata, J. Enzyme Inhib. Med. Chem., 2010, 25, 406-413.

39 J. B. Harborne, Phytochemistry, 1975, 14, 1147-1155.

40 A. Murray, M. Faraoni, M. Castro, N. Alza and V. Cavallaro, Curr. Neuropharmacol., 2013, 11, 388-413.

41 J. H. Kim, S.-H. Lee, H. W. Lee, Y. N. Sun, W.-H. Jang, S.-Y. Yang, H.-D. Jang and Y. H. Kim, Int. J. Biol. Macromol., 2016, 91, 1033-1039.

42 H. Moini, Q. Guo and L. Packer, J. Agric. Food Chem., 2000, 48, 5630-5639.

43 M. Ozyurek, B. Bektasoglu, K. Guclu and R. Apak, Anal. Chim. Acta, 2009, 636, 42-50.

44 A. Fais, B. Era, S. Asthana, V. Sogos, R. Medda, L. Santana, E. Uriarte, M. J. Matos, F. Delogu and A. Kumar, Int. J. Biol. Macromol., 2018, 120, 1286-1293.

45 A. Grosdidier, V. Zoete and O. Michielin, Nucleic Acids Res., 2011, 39, W270-W277.

46 R. A. Laskowski and M. B. Swindells, J. Chem. Inf. Model., 2011, 51, 2778-2786. 